\title{
Vertical Meandering Approach for Antenna Size Reduction
}

\author{
Li Deng, ${ }^{1,2}$ Shu-Fang Li, ${ }^{1}$ Ka-Leung Lau, ${ }^{2}$ and Quan $X^{2} e^{2,3}$ \\ ${ }^{1}$ Key Laboratory of Universal Wireless Communication, Beijing University of Posts and Telecommunications, \\ Beijing 100876, China \\ ${ }^{2}$ Shenzhen Research Institute, City University of Hong Kong, Shenzhen 518057, China \\ ${ }^{3}$ State Key Laboratory of Millimeter Waves, City University of Hong Kong, Kowloon, Hong Kong
}

Correspondence should be addressed to Li Deng, kingdl2001@163.com

Received 15 July 2011; Accepted 30 September 2011

Academic Editor: Ahmed A. Kishk

Copyright () 2012 Li Deng et al. This is an open access article distributed under the Creative Commons Attribution License, which permits unrestricted use, distribution, and reproduction in any medium, provided the original work is properly cited.

A novel vertical meandering technique to reduce the lateral size of a planar printed antenna is presented. It is implemented by dividing a conventional spiral patch into a different number of segments and placing them on different sides of the microwave substrate with vias as the connections. To confirm the validity of this technique, measured electrical performance and radiation characteristics of five antennas with different numbers of segments are compared. The smallest antenna is reduced in size by $84 \%$ when compared with the conventional printed spiral antenna.

\section{Introduction}

The topic of antenna miniaturization has been a subject of interest for more than half a century, but in recent years, it has attained significant attention because of an exorbitant demand for mobile wireless communication systems. The need for antenna miniaturization stems from the fact that most mobile platforms have a limited space for all of the required antennas in ever-increasing wireless systems. Miniature antennas are in high demand, since the antenna size often imposes a significant limitation on the overall size of a portable wireless system.

An example of a miniaturized antenna is a meandered antenna where a half wavelength dipole is made compact by meandering the wire [1]. A similar approach can be applied to design a meander-type slot antenna [2]. Meandering the excited patch surface current paths in the microstrip antenna's radiating patch is also an effective method for achieving a lowered fundamental resonant frequency for the microstrip antenna [3-7]. On the other hand, meandered antennas are very hard to match to a $50 \mathrm{Ohm}$ line. This difficulty is due to the fact that the radiation of almost in-phase electric currents flowing in opposite directions on closely spaced wires or patches tends to cancel each other in the far-field region. This cancellation renders a considerable portion of opposing currents ineffective as far as radiation efficiency is concerned and leads to a very low radiation resistance that might have increased using a twostrip meandered line [8]. Consequently, these antennas are difficult to match, and yet require a very low temperature of operation to control material losses [9].

The technique for lengthening the current path mentioned above is almost based on a coplanar or single-layer microstrip structure. The current path lengthening for a fixed patch antenna can also be obtained by using vertical meandering technique. By vertical meandering, we can use the area of substrate more efficiently. Furthermore, we can reduce the cancellation in the above-mentioned conventional meandering techniques effectively by rational design of the vertical meandering lines.

In this paper, a novel vertical meandering technique aimed at reducing the size of a printed spiral patch antenna is proposed. By utilizing this technique on a single turn conventional printed spiral patch antenna, its resonant frequency can maximally drop from $1.53 \mathrm{GHz}$ to $0.61 \mathrm{GHz}$, which is $60 \%$. Therefore, the antenna size can be reduced by $84 \%$. The antenna finds a reasonable impedance bandwidth $(\mathrm{S} 11 \leq-10 \mathrm{~dB})$ of $2.6 \%$. 


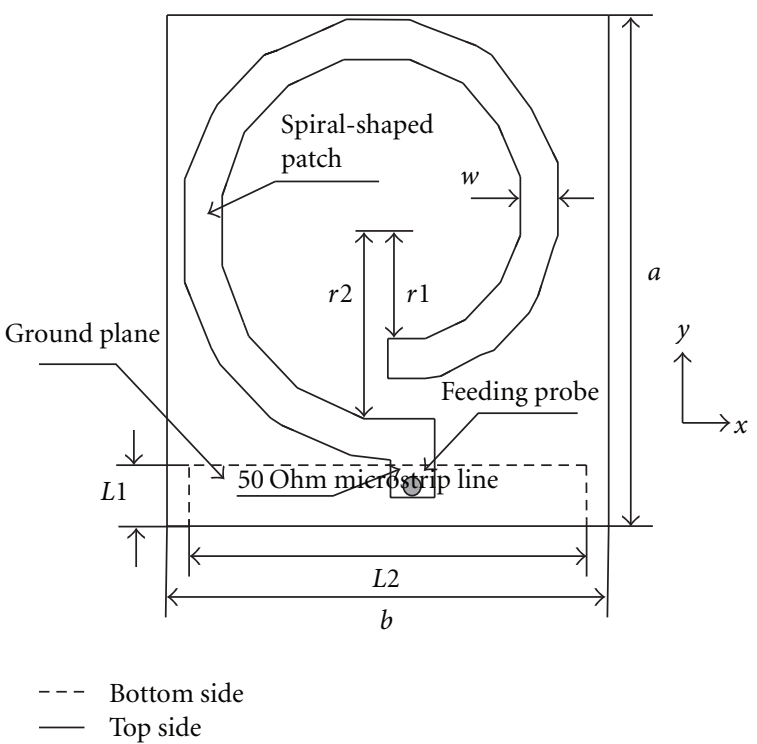

(a)

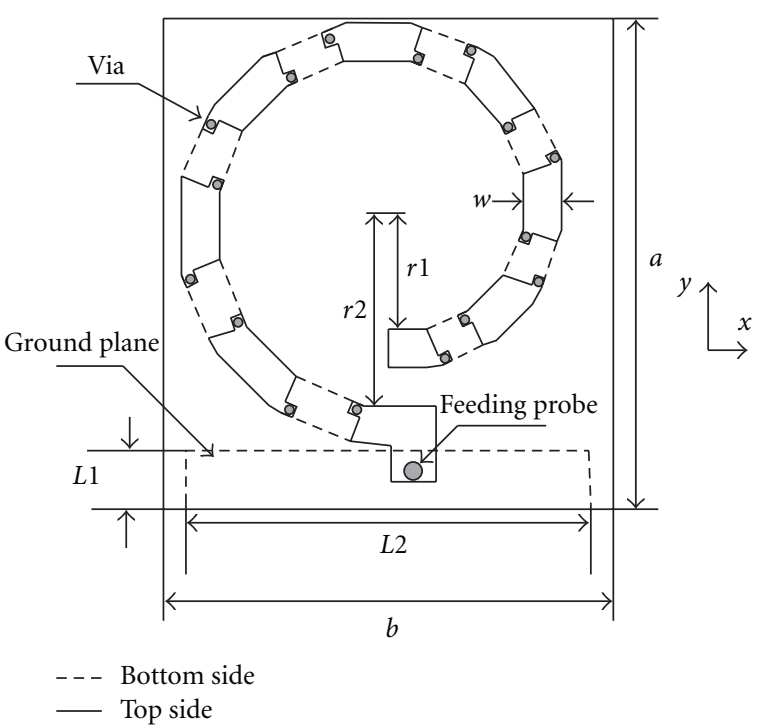

(b)

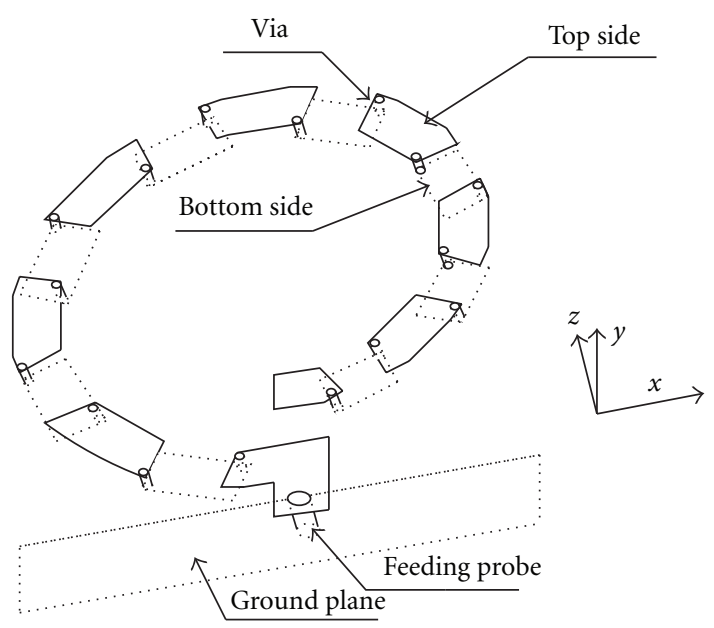

(c)

Figure 1: (a) Top view of the conventional printed spiral antenna. (b) Top view of the novel printed spiral antenna. (c) 3D view of the novel structure.

\section{Antenna Structure}

Figure 1(a) shows the geometry of a conventional printed spiral antenna. It consists of a small rectangular ground plane etched on the bottom side of a F4B substrate, which has thickness of $2 \mathrm{~mm}$ and dielectric constant of 2.65. A single spiral-shaped patch and a $50 \Omega$ microstrip line are printed on the top side of the same substrate. This line is coaxial fed by a $50 \Omega$ SMA (SubMiniature version A) connector underneath the ground plane. The feeding probe has radius of $0.5 \mathrm{~mm}$. In order to reduce the lateral size of this antenna, a novel technique is applied. This technique is implemented by separating the conventional spiral patch in Figure 1(a) into a different number of segments (denoted by $N$ ). Then, half of these segments are moved to the bottom side of the microwave substrate. Finally, vertical conducting vias are used to connect the corners of the segments on the top and bottom layers together (as is well known, the vias are used widely in PCB for connections between layers, it is easy to fabricate). For maximization of the size reduction, the vertical vias should be staggered arranged. Staggered vias at the cross-corners can make the distance of two adjacent vias larger than center arranged vias. Thus, staggered arranged vias have smaller coupling capacitances than center arranged vias; then the effect of cancellation to the inductances is smaller than center arranged vias too. Therefore, for the whole antenna with the same length, the staggered vias at the cross-corners can have larger inductances than center arranged vias, and larger inductance leads to larger size reduction. The proposed antenna structure is depicted in 
Figures 1(b) and 1(c). It is identical to the conventional spiral antenna in Figure 1(a) when $N$ is equal to 1 . Each via has the same radius of $0.3 \mathrm{~mm}$. For the proposed antenna, the physical length of the current flowing path is increased with the number of segments. Therefore, its resonant frequency is decreased with the value $N$. As a result, a larger $N$ can achieve a smaller antenna size. To prove the effectiveness of the proposed size reduction technique, five printed spiral antennas with $N$ equals to $1,16,32,64$, and 128 are fabricated and tested. The other parameters of the antennas are: $r 1=$ $15 \mathrm{~mm}, r 2=25 \mathrm{~mm}, w=5 \mathrm{~mm}, L 1=7 \mathrm{~mm}, L 2=86 \mathrm{~mm}$, $a=70 \mathrm{~mm}, b=92 \mathrm{~mm}$.

\section{Results and Analysis}

In this paper, we make a fully simulation through a MoM(Method of Moments-) based software, IE3D. Figure 2 shows the simulated input return loss of these antennas. It is clearly seen that the antenna is resonant at $1.49 \mathrm{GHz}$ when $N=$ 1. If $N$ is increased to $16,32,64$, and 128 , the resonance frequency is reduced to $1.31 \mathrm{GHz}, 1.14 \mathrm{GHz}, 0.89 \mathrm{GHz}$, and $0.61 \mathrm{GHz}$, respectively. Therefore, the suppression in resonant frequency is $12.1 \%, 23.5 \%, 40.3 \%$, and $59.1 \%$, respectively. The reduction in patch size is $22.7 \%, 41.5 \%$, $64.3 \%$, and $85.5 \%$. The impedance bandwidths (S11 $\leq$ $-10 \mathrm{~dB})$ of these five antennas are $12.3 \%, 11.6 \%, 8.7 \%$, $5.08 \%$, and $2.3 \%$, respectively.

Figure 3 shows the photograph of these five antennas; they have the same structures as the simulated structures. The antenna's performance is measured by the E5071C Network Analyzer and the Near-Field Antenna Measurement System, Satimo. Figure 4 shows the measured input return loss of these antennas. It can be seen that the antenna is resonant at $1.53 \mathrm{GHz}$ when $N=1$. If $N$ is increased to 16,32 , 64 , and 128 , the resonance frequency is reduced to $1.37 \mathrm{GHz}$, $1.2 \mathrm{GHz}, 0.92 \mathrm{GHz}$, and $0.61 \mathrm{GHz}$, respectively. Therefore, the reduction in resonant frequency is $10 \%, 22 \%, 40 \%$, and $60 \%$, respectively. The reduction in patch size is $19 \%$, $39 \%, 64 \%$, and $84 \%$. The impedance bandwidths (S11 $\leq$ $-10 \mathrm{~dB}$ ) of these five antennas are $14 \%, 12 \%, 9 \%, 5.1 \%$, and $2.6 \%$, respectively. In summary, the percentage size reduction for the proposed antenna is increased with the number of segments used. More reduction in size is achieved at the cost of narrower bandwidth. This is expected because the bandwidth of an antenna is related to the electrical volume of its radiation.

Figure 5(a) to Figure 5(d) show the measured radiation patterns of the proposed antennas with $N=1$ to 64 in plane $\phi=0^{\circ}$ and $\phi=90^{\circ}$. Because of the small ground plane, the radiation patterns for the antennas are bidirectional. Patterns for the antenna with $N=128$ are not provided since the lowest measurement frequency of our Near-Field Antenna Measurement System $(0.8 \mathrm{GHz})$ is higher than the resonant frequency $(0.61 \mathrm{GHz})$ of this antenna. The gain at the resonance frequency $1.53 \mathrm{GHz}$ is $1.2 \mathrm{dBi}$. When $N$ is increased to 16,32 , and 64 , the gain is reduced to $0.4 \mathrm{dBi}$, $-0.45 \mathrm{dBi}$, and $-1.5 \mathrm{dBi}$. Therefore, increasing the number of segments will decrease the antenna gain. This is also

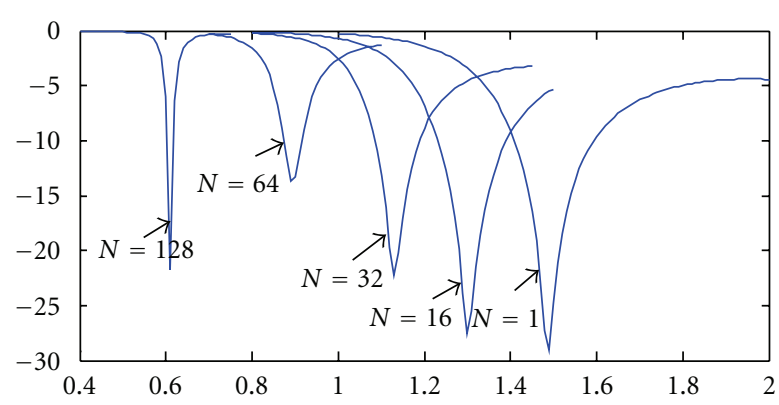

Figure 2: Simulated input return loss of these antennas.

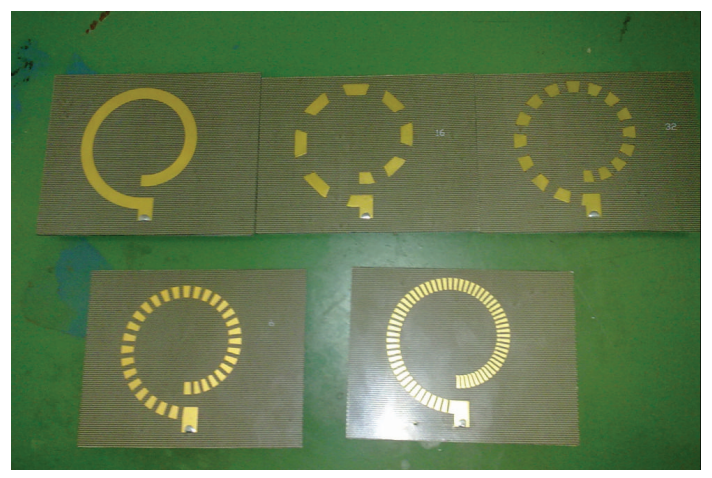

Figure 3: Photograph of these five antennas.

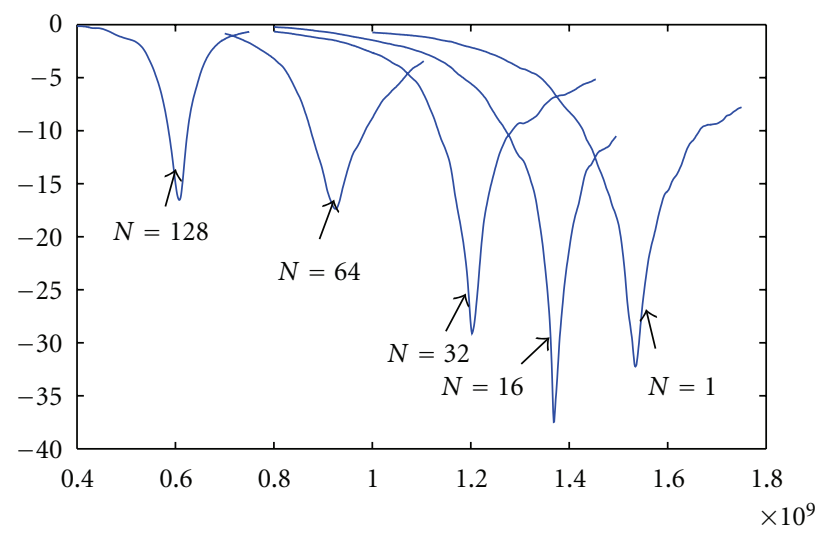

FIGURE 4: Measured input return loss of these antennas.

expected because the radiation efficiency of an antenna is dropped with its electrical radiation area [10]. We can see from Figure 5(b) to Figure 5(d) that copolarization and cross-polarization components are very close to each other. The reason is that the increasing number of vias leads to the inconsistencies in the structure borders and makes more cross-polarization components which cannot be canceled out. This will be solved in future research.

\section{Conclusion}

A novel vertical meandering technique to reduce the lateral size of a printed spiral antenna is proposed. Five printed 


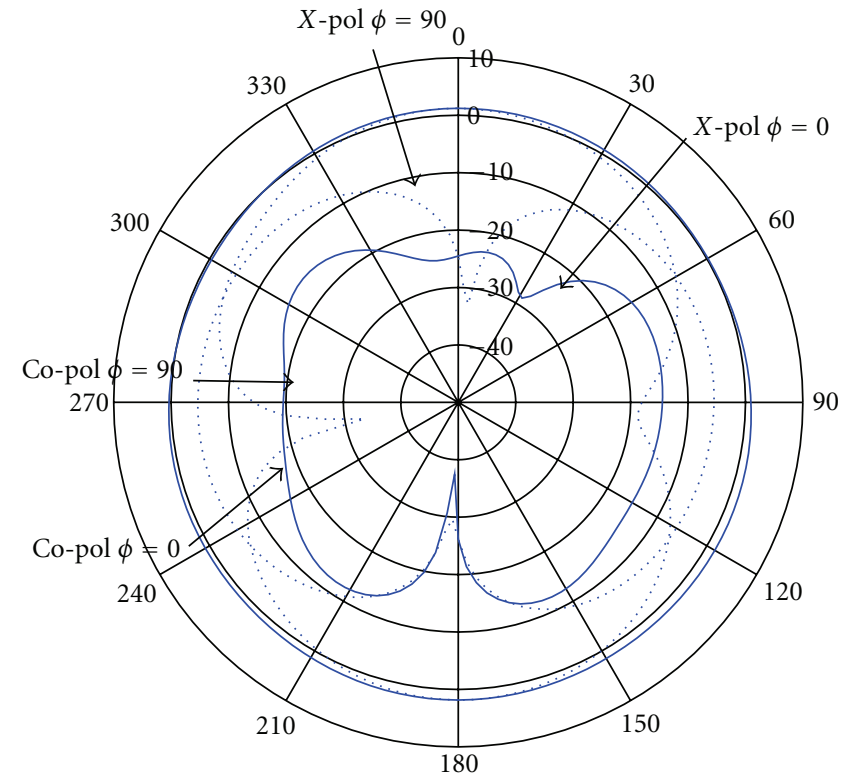

(a)

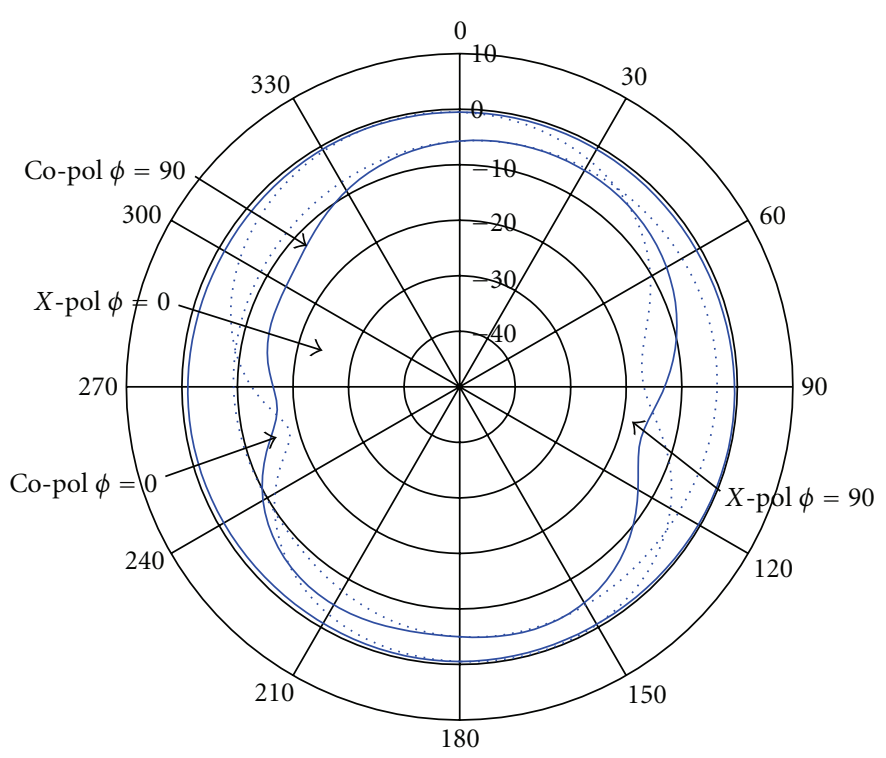

(c)

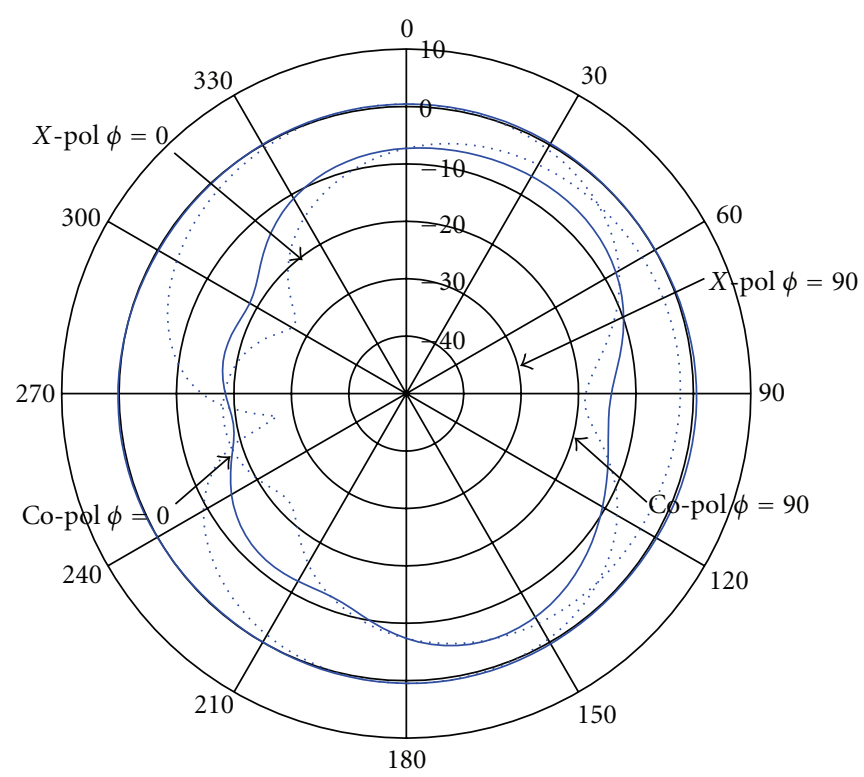

(b)

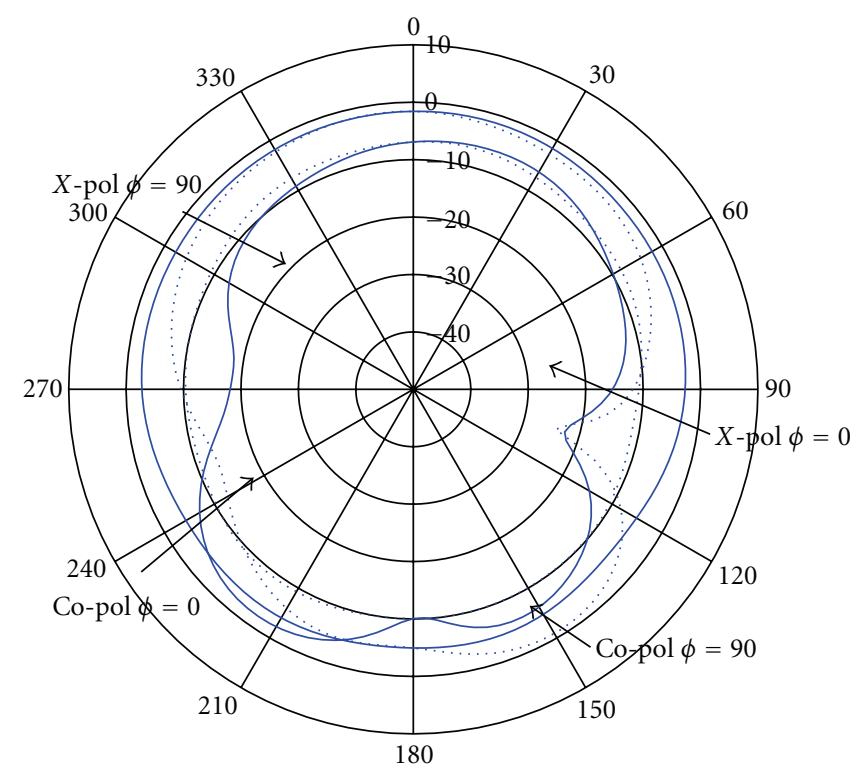

(d)

FIGURE 5: (a) Measured radiation patterns with $N=1$. (b) Measured radiation patterns with $N=16$. (c) Measured radiation patterns with $N=32$. (d) Measured radiation patterns with $N=64$.

spiral antennas with a different number of segments are constructed, tested, and analyzed. According to the results achieved, it is evident that the size of the antenna can be significantly reduced by increasing its number of segments.

\section{Acknowledgments}

The authors wish to thank Dr. Ji Li and Weijun Hong for their helpful comments on the work presented in this paper. This work was supported in part by Shenzhen Science and Technology Planning Project for the Establishment of Key Laboratory in 2009 (CXB 200903090021A) and Hi-Tech
Research and Development Program of China (863 Program, no. 2006AA04A106).

\section{References}

[1] J. Rashed and C. T. Tai, "A new class of resonant antennas," IEEE Transactions on Antennas and Propagation, vol. 39, no. 9, pp. 1428-1430, 1991.

[2] J. M. Kim and J. G. Yook, "Compact mender-type slot antennas," in Proceedings of the IEEE Antennas and Propagation Society International Symposium, vol. 2, pp. 724-727, Boston, Mass, USA, July 2001. 
[3] S. Dey and R. Mittra, "Compact microstrip patch antenna," Microwave and Optical Technology Letters, vol. 13, no. 1, pp. 12-14, 1996.

[4] K. L. Wong, C. L. Tang, and H. T. Chen, "A compact meandered circular microstrip antenna with a shorting pin," Microwave and Optical Technology Letters, vol. 15, no. 3, pp. 147-149, 1997.

[5] C. K. Wu, K. L. Wong, and W. S. Chen, "Slot-coupled meandered microstrip antenna for compact dual-frequency operation," Electronics Letters, vol. 34, no. 11, pp. 1047-1048, 1998.

[6] J. H. Lu and K. L. Wong, "Slot-loaded, meandered rectangular microstrip antenna with compact dual-frequency operation," Electronics Letters, vol. 34, no. 11, pp. 1048-1050, 1998.

[7] J. George, M. Deepukumar, C. K. Aanandan, P. Mohanan, and K. G. Nair, "New compact microstrip antenna," Electronics Letters, vol. 32, no. 6, pp. 508-509, 1996.

[8] K. Noguchi, N. Yasui, M. Mizusawa, S. I. Betsudan, and T. Katagi, "Increasing the bandwidth of a two-strip meanderline antenna mounted on a conducting box," in Proceedings of the IEEE Antennas and Propagation Society International Symposium, pp. 112-115, July 2001.

[9] H. Chaloupka, N. Klein, M. Peiniger, H. Piel, A. Pischke, and G. Splitt, "Miniaturized high-temperature superconductor microstrip patch antenna," IEEE Transactions on Microwave Theory and Techniques, vol. 39, pp. 1513-1521, 1991.

[10] L. J. Chu, "Physical limitations of omni-directional antennas," Journal of Applied Physics, vol. 19, no. 12, pp. 1163-1175, 1948. 

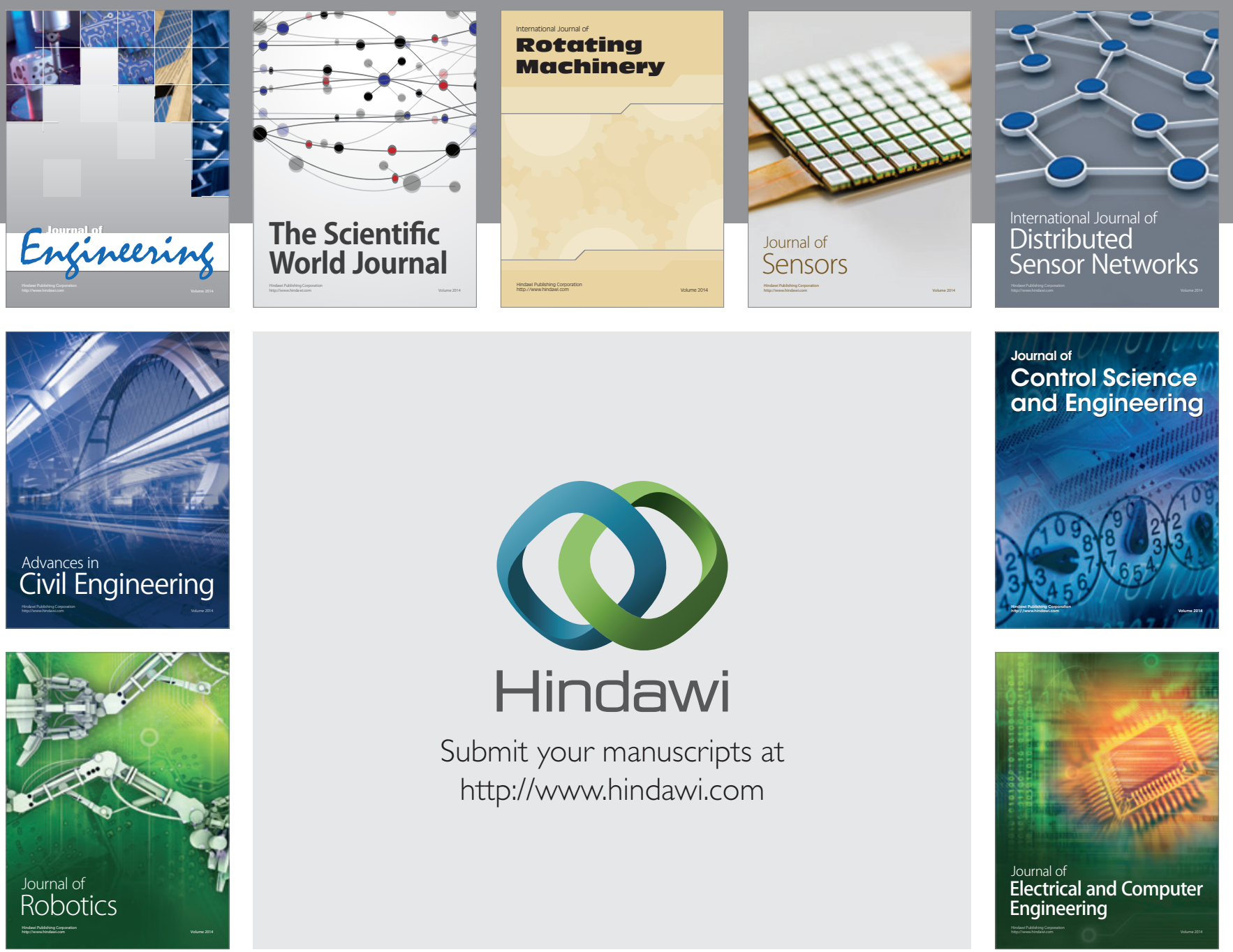

Submit your manuscripts at

http://www.hindawi.com
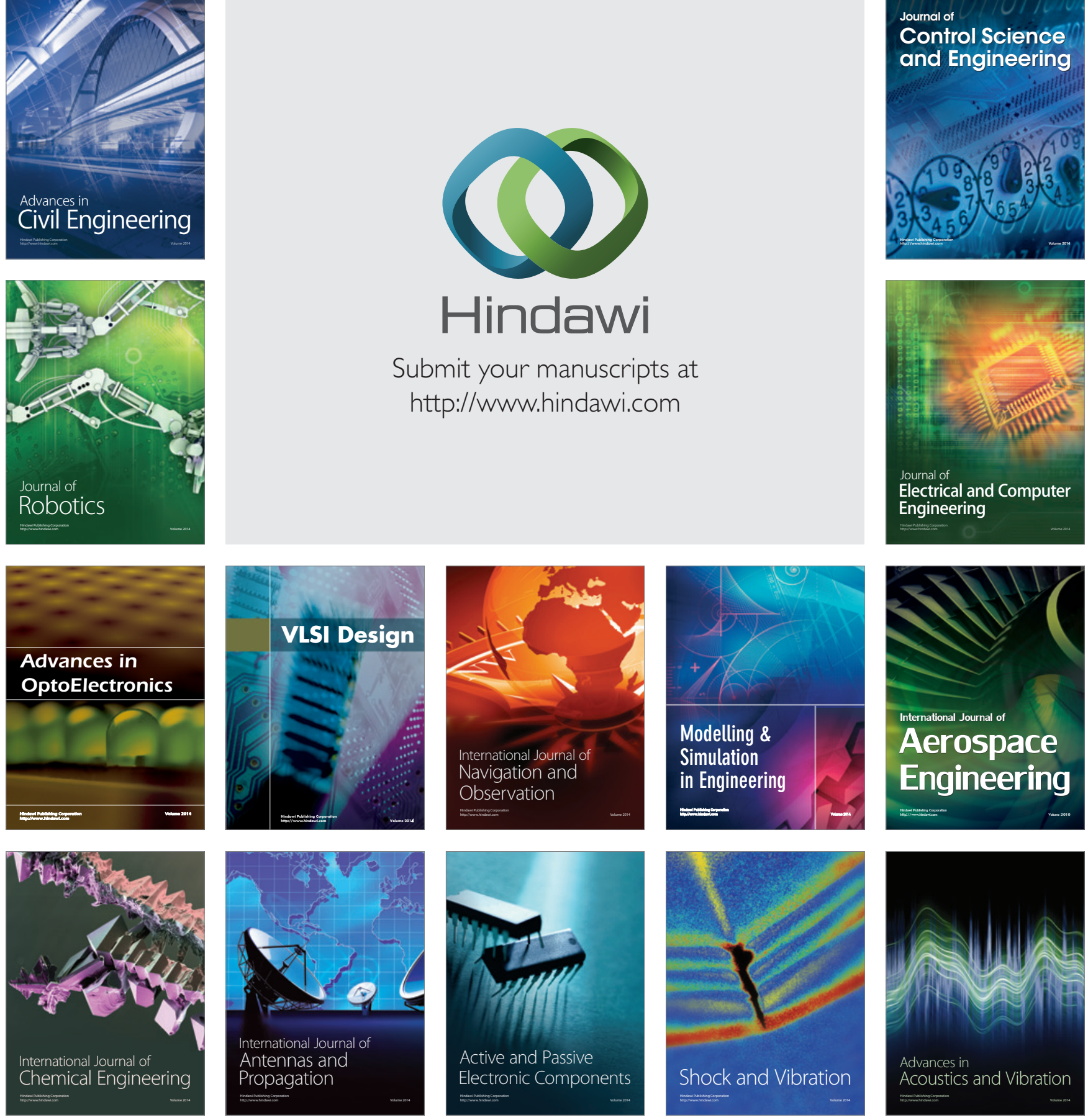